\title{
Bryophytes of Libya. I. Hepatophyta: an Annotated Checklist
}

\author{
S.G. Youssef ${ }^{\#}$ \\ Botany Department, Faculty of Science, BenhaUniversity, Benha, Egypt.
}

\begin{abstract}
7 HE list of Hepatophyta in Libya includes 29 taxa in 15 genera, 14 families, 7 orders and two classes. Anthocerotae is represented by one species while Hepaticae by 27 species. No species were reported after 1968; recent collections are therefore necessary. Riccia is dominant being represented by 12 species. Synonyms are given in a separate list.
\end{abstract}

Keywords : Hepatophyta, Checklist, Libya.

\section{Introduction}

Research work on Hepatophyta (Hepatics and Anthocerotes) of Libya (Fig. 1) started more than one century ago. However the number of papers published is relatively small; being published at long irregular intervals as follows: Ascherson (1881), Baccarini (1913), Massalongo (1914), Zodda (1914), Pampanini (1917), Zodda (1926), Pampanini (1931), Maire \& Weiler(1939), Bizot \& Lauriol (1950), Jovel-Ast (1956), Rungby (1962), Jovel-Ast \& Bisscher (1968) and Ros et al. (1999, 2007). Those above authors reported 29 taxa (27 species, one subspecies and one varity) in 15 genera, 14 families, seven orders and two classes as comes below in the Table 1.

Dates of collection of the 29 taxa are as expressed briefly in the legend above table one. The available literature (Pampanini, 1931) does not give more precise dates and gives sites of collection (Fig.1) of only the following taxa: Riccia ciliata, Benghazi; R. lamellosa, Benghazi; $R$. nigrella, Benghazi; Pellia endiviifolia, Cavra (Wadi Belgadir) and Wadi Marsa Susa; Plagiochasma rupestre, Cavra (Wadi Samber) and Targionia hypophylla, Cerene.

\section{Annotations} listed:

Based on the list above the following annotations were

1- Class Anthocerotae is represented in Libya by one family and one species while class Hepaticae by 13 families and 27 species.

2- Marchantiales is represented by 6 families, Jungermanniales and Sphaerocarpales by two families each while Fossombroniales,Lunulariales and Pelliales by one family each.
3- All families of Hepaticae are represented by one genus each, only Aytoniaceae is represented by two genera.

4- Genus Riccia is dominant being represented by 12 species, while the majority of the other genera are represented by only one species.

5- Twenty-five species are reported during or after 1962 (symbol •), while other symbols [(•), o, (o) and +] represent one species each.

6- Porella cordaeana (Huebener) Moore and Plagiochila africana Steph.are not given in the above list because they were erroneously reported from Libya (Rungby, 1962; Ros et al., 1999 and Söderström et al., 2002).

It has to be mentioned in conclusion and based on the given references that no hepatophytes collections were made after 1968, which means that the present list represents the hepatophytes of Libya since about 50 years ago. No need to say that recent collections are therefore necessary from this country.

\section{Acknowledgement}

All thanks, gratitude and appreciation to Dr. El- Saadawi Professor of Botany at Ain Shams University for useful criticism and kind help, may God reward him.

\section{Synonyms}

Synonyms mentioned below are only those used in publications related to Libya.

Fossombronia husnotii Corb. = Fossombronia caespitiformis subsp. multispira (Schiffn.) J.R. Bray and D.C. Cargill in Stotler et al.

Pellia fabbroniana Raddi = Pellia endiviifolia Dicks.) Dumort

Plagiochasma algericum Steph. = Plagiochasma rupestre (J. R. Forst. \& G. Forst.) Steph.

Riccia trichocarpa M. Howe = Riccia crinita Taylor

\#Corresponding author email: msmyes68@yahoo.com. 


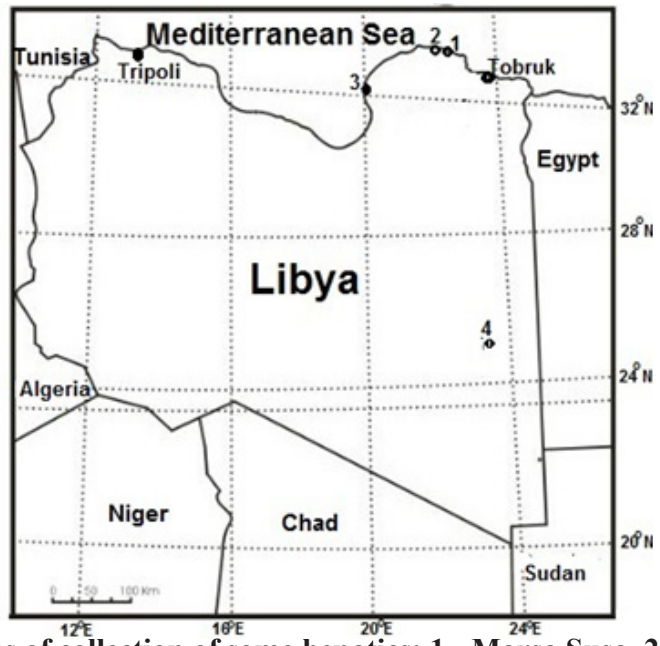

Fig.1. Map of Libya and sites of collection of some hepatics; 1- Marsa Susa, 2- Cerene, 3-Benghazi and 4- Cavra.

TABLE 1. Names of the 29 Hepatophyta taxa, families and orders they belong to and other relevant information, where $0=$ Report based on collections published before $1962, \bullet=$ Report based on at least one collection published during or after 1962, $(0)=$ Doubtful report based on collections published before 1962, $(\bullet)=$ Doubtful report based on at least one collection published during or after 1962, += Report without precise collection data or locality. These symbols are used here exactly as given in Roset al. (2007) for the purpose of giving herefurther meaningful annotations.

\begin{tabular}{|c|c|c|c|}
\hline $\begin{array}{l}\text { Class } \\
\text { Order }\end{array}$ & Family & Species & Symbol \\
\hline \multicolumn{4}{|l|}{ Anthocerotae } \\
\hline \multicolumn{4}{|l|}{ Hepaticae } \\
\hline \multirow{2}{*}{ Fossombroniales } & \multirow{2}{*}{ Fossombroniaceae } & $\begin{array}{l}\text { Fossombronia caespitiformis subsp. Caespitiformis De } \\
\text { Not. ex Rabenh. }\end{array}$ & • \\
\hline & & $\begin{array}{l}\text { F. caespitiformis subsp. multispira (Schiffn.) J R.Bray \& } \\
\text { D.C. Cargill. }\end{array}$ & o \\
\hline \multirow{2}{*}{ Jungermanniales } & Arnelliaceae & Southbya nigrella (De Not.) Henriq. & • \\
\hline & Cephaloziellaceae & Cephaloziella baumgartneri Schiffn. & • \\
\hline Lunulariales & Lunulariaceae & Lunularia cruciata (L.) Lindb. & • \\
\hline \multirow{19}{*}{ Marchantiales } & \multirow[t]{2}{*}{ Aytoniaceae } & Plagiochasma rupestre (J. R. Forst. \& G. Forst.) Steph. & (o) \\
\hline & & Reboulia hemisphaerica (L.) Raddi & $\bullet$ \\
\hline & Cleveaceae & Athalamia spathysii (Lindenb.)S.Hatt. & • \\
\hline & Conocephalaceae & Conocephalum conicum (L.) Dumort. & $(\bullet)$ \\
\hline & Oxymitraceae & Oxymitra incrassata (Brot.) Sérgio \& Sim-Sim & • \\
\hline & \multirow{12}{*}{ Ricciaceae } & Riccia atromarginata Levier & • \\
\hline & & R. cavernosa Hoffm. & $\bullet$ \\
\hline & & R. ciliata Hoffm. & • \\
\hline & & R. crinita Taylor & • \\
\hline & & R. crustata Trab. & $\bullet$ \\
\hline & & R. crystallina L. emend. Raddi & $\bullet$ \\
\hline & & R. gougetiana Durieu \& Mont. & + \\
\hline & & R. lamellosa Raddi & $\bullet$ \\
\hline & & R. michelii Raddi & $\cdot$ \\
\hline & & R. nigrella DC. & $\cdot$ \\
\hline & & R. sorocarpa Bisch.var. sorocarpa. Bisch. & $\cdot$ \\
\hline & & R. trabutiana Steph. & $\bullet$ \\
\hline & \multirow{2}{*}{ Targioniaceae } & Targionia hypophylla L. & $\bullet$ \\
\hline & & T. lorbeeriana Müll. Frib. & $\cdot$ \\
\hline Pelliales & Pelliaceae & Pellia endiviifolia (Dicks.) Dumort. & $\cdot$ \\
\hline \multirow{3}{*}{ Sphaerocarpales } & Riellaceae & Riella cyrenaica Maire & $\cdot$ \\
\hline & \multirow{2}{*}{ Sphaerocarpaceae } & Sphaerocarpos michelii Bellardi & • \\
\hline & & S. texanus Austin & • \\
\hline
\end{tabular}




\section{References}

Ascherson, P. (1881) Plantarum Africae septentrionalis mediae hucusque cognitarum Botanisches Centralblatt, 8, 278-287.

Baccarini, P. (1913) Sede di Firenze. Adunanza dell'8 Marzo 1913. Bollettinodella Societd Botanica Italiana, 39-40.

Bizot, M. and Lauriol, J. (1950) Bryophyta. In : "Etudes sur la Flore et la Vegetation du Tibesti" Maire R \& Monod T.(Ed).. Memoires de l'Insti-tut d 'Afrique Noire, 8, 22-23.

Jovet-Ast, S. (1956) Hépatiques Marocaines. - II. Bulletin de la Société des Sciences Naturelles et Physiques du Maroc 36, 43-60.

Jovet-Ast, S. and Bischler, H. (1968) Les hépatiques de Libye. Enumerationnotes ecologiquesetbiogeographiques. Revue bryologique et lichénologique, 36, 245-291.

Maire, R. and Weiler, M. (1939) Contributions a l'etudede la Flore de Libye. Bulletin de ta Societed' Histoire Naturelle de I'Afrique du Nord, 30, 255-314.

Massalongo, C. (1914) Hepaticae Tripolitanaea R. Pampanini anno 1913 lectae. Nuovo Giornale Botanicoltaliano, 21, 10.

Pampanini, R. (1917) Plante di Bengasi e del suoTerritorioraccolte dal Rev. P.D. Vito Zanondella Missionedel PP. Giuseppini al Fuehat. Nuovo Giornale Botanico taliano, 24, 113171.
Pampanini, R. (1931) Prodromodella Flora Cirenaica. Forli.

Ros, R.M., Cano, M.J. and Guerra, J. (1999) Bryophyte checklist of Northern Africa. Journal of Bryology, 21, 207-244.

Ros, R.M., Mazimpaka, V., Abou-Salama, U., Aleffi, M., Blockeel, T.L., Bruguès, M., Cano, M.J., Cros, R.M., Dia, M.G., Dirkse, G.M., El Saadawi, W., Erdaa, A., Ganeva, A., Gonzàlez-Manecebo, J.M., Herrnstadt, I., Khalil, K., Kürschner, H., Lanfranco, E., Losada-Lima, A., Refai, M. S., Rogrígueznuñez, S., Sabovliević, M., Sérgio, C., Shabbara, H.M., Sim-Sim, M. and Söderström, L. (2007) Hepatics and Anthocerotes of the Mediterranean, an annotated checklist. Cryptogamie, Bryologie, 28, 351-437.

Rungby, S. (1962) A contribution to the bryophytic flora of Spain and Morocco, especially the area between Gandia and Alcoy. Botaniska Notiser, 115, 61-64.

Söderström, L., Urmi, E. andVàña, J. (2002) Distribution of Hepaticae and Anthocerotae in Europe and Macaronesia. Lindbergia, 27, 3-47.

Zodda, G. (1914) Manipolo di Briofitedella Tripolitania. Bolletinodell'OrtoBotanicodella $R$. Universita di Napoli, 4, 205-209.

Zodda, G.(1926)DialcuneBriofitedellaCirenaicaraccolte dal Prof.Cavara. Bolletinodell'OrtoBotanicodella R. Universita di Napoli, 8, 235-238.

Received :10/1/2017;

Accepted :26/ 2 /2017

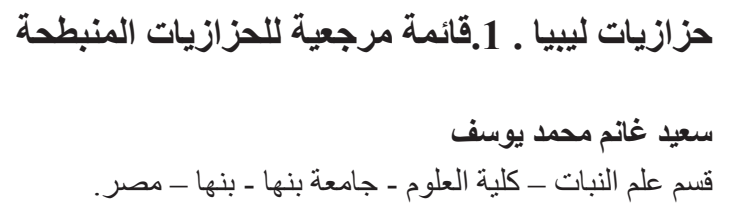

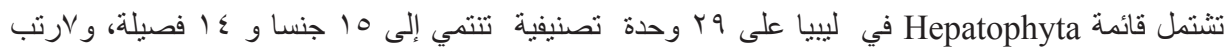

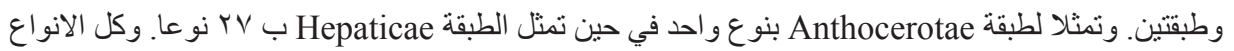

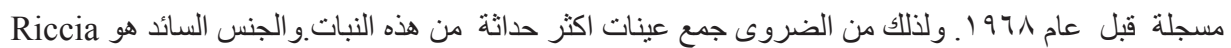

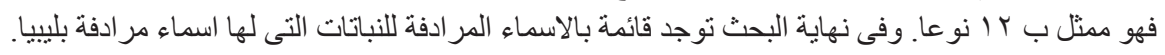



